\section{Jährliche Mammografie - häufig falsche Befunde}

\begin{abstract}
Wird bei Frauen ab 40 Jahren jedes Jahr statt alle zwei Jahre die Brust per Mammografie untersucht, so führt das nicht unbedingt zu einer früheren Krebsdiagnose. In einer aktuellen Studie stieg damit lediglich die Zahl der positiven Befunde, bei denen sich der Tumorverdacht als falsch erwies.
\end{abstract}

$\int$ edes edes Jahr oder nur alle zwei Jahre die Brust röntgen? Welches der beste Weg ist, möglichst frühzeitig Brustkrebs zu entdecken, darüber streiten sich noch die Experten. Mit dem jährlichen Screening, so die Hoffnung, lassen sich Tumoren in einem früheren Stadium aufspüren. In Kauf nehmen müssen Frauen dafür allerdings, dass auch öfter mal eine Untersuchung einen Krebsverdacht weckt, der sich in der weiteren Diagnostik als falsch erweist. Die Folge sind viele Zusatzuntersuchungen, mitunter schmerzhafte Biopsien und die Angst, an Krebs erkrankt zu sein.

Ein Team um Rebecca Hubbard aus Seattle in den USA hat nun Daten von knapp 170.000 Frauen ausgewertet, die zwischen 1994 und 2006 erstmals an einem Mammografie-Screening teilnahmen. Die Forscher verglichen dabei über einen Zeitraum von zehn Jahren hinweg die Raten von falsch positiven Befunden und den Anteil der Frauen mit fortgeschrittenem Mammakarzinom bei einjähriger und zweijähriger Mammografie.

Die Ergebnisse: Die Wahrscheinlichkeit, innerhalb von zehn Jahren mindestens einen falsch positiven Befund zu erhalten, betrug bei jährlichem Screening $61,3 \%$, bei zweijährigem 41,6\%. Dabei spielte es praktisch keine Rolle, ob die Frauen im Alter von 40 Jahren mit der Mammografie anfingen, wie es viele Experten raten, oder erst ab 50 Jahren, wie es in Deutschland auch von den Kassen bezahlt wird. Mit der jährlichen Mammografie wurde bei $7 \%$ der Frauen ab 40 Jahren aufgrund falsch positiver Befunde auch eine Biopsie veranlasst, mit zweijährigem Screening nur bei $4,8 \%$. Bei den Frauen ab 50 Jahren war der Unterschied mit 9,4 versus $6,4 \%$ ähnlich deutlich.

Eine Krebsdiagnose wurde bei knapp 4.500 der Frauen gestellt (2,6\%). Von den krebskranken Frauen im Alter von 40 bis 49 Jahren hatten mit jährlichem Screening bei der Diagnose 21,6\% einen Tumor im späten Stadium, mit zweijährigem Scree- ning waren es $24,6 \%$ - ein Unterschied von 3,3 Prozentpunkten. Bei den älteren Frauen lagen diese Werte mit 19,6 versus $21,9 \%$ ebenfalls etwas auseinander $(2,3$ Prozentpunkte), allerdings waren die Unterschiede in beiden Altersgruppen nicht signifikant.

Fazit: Lässt sich daraus schließen, dass eine jährliche Mammografie verglichen mit einer zweijährigen nur mehr Kosten, Ängste und unnötige Zusatzdiagnostik verursacht? Nicht unbedingt. Trotz der großen Zahl von teilnehmenden Frauen war die Zahl solcher mit späten Tumorstadien zu klein, um daraus bei den erfassten geringen Unterschieden Signifikanzen abzuleiten. Um die Frage zu klären, bräuchte man also noch größere Datenmengen - und damit noch größere Studien, so die Autoren. Thomas Müller

Hubbard RA et al. Cumulative Probability of false-positive recall or biopsy recommendation after 10 years of screening mammography. Ann Intern Med. 2011;155(8):481-92.

Kommentar von Prof. Schreer: Die vorliegende Studie spiegelt die aktuelle amerikanische Screening-Situation wider: Zurzeit liegen zwei sehr unterschiedliche Empfehlungen zur Brustkrebs-Früherkennung mit regelmäßiger Mammografie vor. Während die amerikanische Krebsgesellschaft und die amerikanische senologische Gesellschaft die jährliche Mammografie ab dem 40. Lebensjahr empfehlen, spricht sich die die „US Task Force" für ein Mammografie-Screening ab dem 50. Lebensjahr in zweijährlichem Abstand aus. Dieser Empfehlung hat sich 2011 auch die $_{\text {"Canadian Task Force on Preventive }}$ Health Care" angeschlossen. Wesentliche Argumente gegen den Beginn mit dem 40. Lebensjahr und jährliche Intervalle sind die geringere Brustkrebs-Inzidenz sowie höhere Abklärungsraten (dichteres Drüsengewebe, geringere Sensitivität der Mammografie) mit entsprechend negativen Auswirkungen für die betroffenen Frauen. Eine zentrale Aufgabe jedes Früherkennungsprogramms ist es, die Balance zwischen akzeptablen Abklärungsraten und höchst möglicher BrustkrebsDetektion in frühen Stadien zu wahren. Während in der vorliegenden Studie in der ersten Runde 16,3\% der Frauen wiedereinbestellt wurde, liegt dieser Wert in unserem Screening bei $7-8 \%$, in der Folge-Runde in den USA bei $9,6 \%$ gegenüber $<5 \%$.Wichtiger als die Recall-Rate ist die nach Abklärung resultierende Biopsie-Rate. Sie liegt mit 2,5\% mehr als doppelt so hoch wie bei uns ( $1 \%$ in der ersten Runde). Auch die falsch positive Biopsie-Rate liegt mit $1 \%$ um etwa das Doppelte höher als in unserem Programm. Einschränkend muss erwähnt werden, dass hier

\section{„Die Studie bestätigt die Notwendigkeit konsequent hoher Qualitätsstandards \\ für Früherkennungsprogramme."}

Frauen zwischen 40 und 59 Jahren eingeschlossen wurden. Auch geht aus dem Bericht nicht klar hervor, ob nur für invasive Karzinome berechnet wurde unter Ausschluss der nicht invasiven, die bei uns enthalten sind. Das ließe noch höhere Abklärungsraten vermuten. Die Studie bestätigte den bekannten Zusammenhang zwischen Expertise und Falsch-Positiv-Rate (FPR). Auch die Hinzuziehung von Voraufnahmen konnte die FPR halbieren, d. h. die Spezifität erhöhen. Die angegeben Zahlen für ein 10-JahresIntervall beruhen nur auf Schätzungen, da $47,7 \%$ nur eine Untersuchung hatten und nur 11,8\% mehr als fünf Untersuchungen. Zweijährliche Intervalle reduzierten die FPR, aber um den Preis der Entdeckung von mehr fortgeschrittenen Karzinomen (3\% für Frauen von 40-49 Jahren und 2,3\% für die 50-59jährigen). Diese Zahlen waren allerdings nicht statistisch signifikant aufgrund zu kleiner Gesamtzahlen. Meines Erachtens bestätigt die vorliegende Studie einmal mehr die Notwendigkeit konsequent hoher Qualitätsstandards bei der Durchführung von Früherkennungsprogrammen, um Nutzen zu optimieren und Schaden zu minimieren.

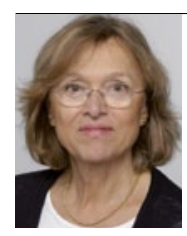

Prof. Dr. med. Ingrid Schreer Mammazentrum, Klinik für Gynäkologie und Geburtshilfe, Universitätsklinikum Schleswig-Holstein, Campus Kiel ischreer@email.uni-kiel.de 